\title{
Early Results from NASA's AsSessment of SATEllite Servicing
}

\author{
Benjamin B. Reed, Jacqueline A. Townsend, Harley A. Thronson, JR., \\ MANSOOR AHMED, ARTHUR O. WhipPle, AND William R. OEgERLE \\ NASA Goddard Space Flight Center \\ Greenbelt MD 20771 \\ USA \\ SPIE TELESCOPES AND INSTRUMENTATION \\ San Diego, CA \\ June 27, 2010 \\ Paper 7731-2
}

\begin{abstract}
Following recommendations by the NRC, NASA's FY 2008 Authorization Act and the FY 2009 and 2010 Appropriations bills directed NASA to assess the use of the human spaceflight architecture to service existing/future observatory-class scientific spacecraft. This interest in satellite servicing, with astronauts and/or with robots, reflects the success that NASA achieved with the Shuttle program and HST on behalf of the astronomical community as well as the successful construction of ISS. This study, led by NASA GSFC, will last about a year, leading to a final report to NASA and Congress in autumn 2010. We will report on its status, results from our March satellite servicing workshop, and recent concepts for serviceable scientific missions.
\end{abstract}

\section{BACKGROUND}

For the past several years, NASA has received formal advice, recommendations, and, more recently, appropriated funding to develop concepts for satellite servicing and assess their value. In 2007, the NASA Advisory Committee Astrophysics Subcommittee recommended that NASA carry out trade studies on "in-space operations [and their] potential for assembly, servicing, and deployment."

A year later in NASA's FY08 Authorization, Congress also declared its interest in the importance of spacecraft servicing: "The [NASA] Administrator shall take all necessary steps to ensure that provision is made in the design and construction of all future observatory-class scientific spacecraft ... for robotic or human servicing and repair ..." [Public Law 110-422 NASA Authorization Act of 2008.]

Subsequently, Congress stressed the importance of linking science with human space flight and directed NASA to take advantage of NASA Goddard Space Flight Center's demonstrated experience, viz., "Therefore, it will be critical that [NASA's human space flight program] demonstrate unique capabilities to maintain synergies between free-flying 
scientific spacecraft and human spaceflight endeavors. Accordingly, the bill provides $\$ 20,000,000$ for NASA to undertake an assessment of the feasibility of using the [human space flight] architecture to service ... observatory-class scientific spacecraft, fully utilizing the [capabilities] for in-space servicing developed by the Goddard Space Flight Center and its private sector partners for the Hubble Space Telescope." [Omnibus Appropriations Act, 2009]

\section{INTRODUCTION}

In response to the request from Congress, the Satellite Servicing Study team was assembled at NASA Goddard Space Flight Center (GSFC) and developed a project plan to poll the space flight community, complete necessary research, and draft a final report that addressed the questions posed. Since the general topic has been studied frequently in the past, it was essential to begin by asking what is already known. Thus, the first step in this plan was the release of a broad Request For Information (RFI) related to on-orbit satellite servicing. The RFI was not intended to lead to procurement; instead, it was an invitation to respond with perspectives on on-orbit satellite servicing. Responses were received from the breadth of stakeholders: academia, industry, government organizations, US and international organizations, satellite builders and users, potential customers, (e.g., science missions, commercial communication satellites, DOD) and potential service providers, large and small commercial ventures producing a fleet of buses or just a specific technology related to satellite servicing.

The study team reviewed the responses and identified promising approaches and technologies to be investigated or presented during the three-day, International Workshop on On-Orbit Satellite Servicing held at the end of March 2010. The majority of the speakers at the workshop were selected from the RFI responses, and the workshop drew an audience of more that 300 attendees. The study team took this opportunity to discuss the ideas in the RFI responses and the workshop presentations, as well as describe the study and invite feedback. The workshop and the presentations are described more fully in http//servicingstudy.gsfonasa.gov/.

Concurrent with the RFI and the Workshop, the team defined the trade space of activities could be called on-orbit satellite servicing. Tasks ranged from simple orbit modification (e.g., circularizing the orbit of a satellite that failed to meet desired orbit) to replacing hardware that was designed to be serviced, to complex unplanned repairs and assembly activities. Once the trade space was defined, the team drafted a suite of notional missions that sampled this trade space, particularly areas that had not already been assessed or accomplished on-orbit.

The notional missions were developed into mission concepts in a collaborative design environment at GSFC's Integrated Design Center (IDC) with the following objectives: 1) to identify major mission drivers and "tall poles," 2) to identify commonalities and key technologies that enable multiple notional missions and, thus, multiple possible servicing architectures, and 3) to equip the study team with information and insight essential to answer the questions that must be answered in the final report. 
It should be noted that the Congressional language focused on identifying elements of the Constellation architecture that enable satellite servicing, such as an airlock for the Orion spacecraft. However, as the study progressed the reality of the Constellation Program waned and the 'Flexible Path' alternative was proposed by the White House Augustine Committee (http/www.nasa.gov/offices/hsf/home/index.html). Since the notional missions were envisioned to identify commonalities and technologies that enable multiple future architectures, we were able to respond to the uncertainties in the NASA human spaceflight program during the course of the study. As a result, some of the notional missions involve Constellation elements, some involve Commercial Orbital Transportation Services (COTS), and some involve sending humans to ever greater distances from the earth (as summarized in the Augustine Committee's 'Flexible Path').

Equipped with the RFI responses, the Workshop proceedings, a year of research, and the notional mission products, the team will draft a final report that addresses the following questions:

1. What accommodations are required within the human flight architecture to enable its use as a servicing provider?

2. What does it mean to make a spacecraft serviceable "to the greatest extent practicable"'?

3. What is the cost-benefit trade for servicing?

4. What are the key enabling technologies and gaps between current TRL and servicing needs?

\section{Trade SPACE}

The term 'satellite servicing' was not defined and, therefore, not restricted in the Congressional language. We believe this was done intentionally to encourage us to adopt a broad definition of the term. The team worked to identify the various parameters of satellite servicing trade space (Figure 1). Once this was done we then set about poring through the historical records to document which portions of the trade space have been investigated and which were unexplored. These two non-intersecting regions are identified in Figure 1. 


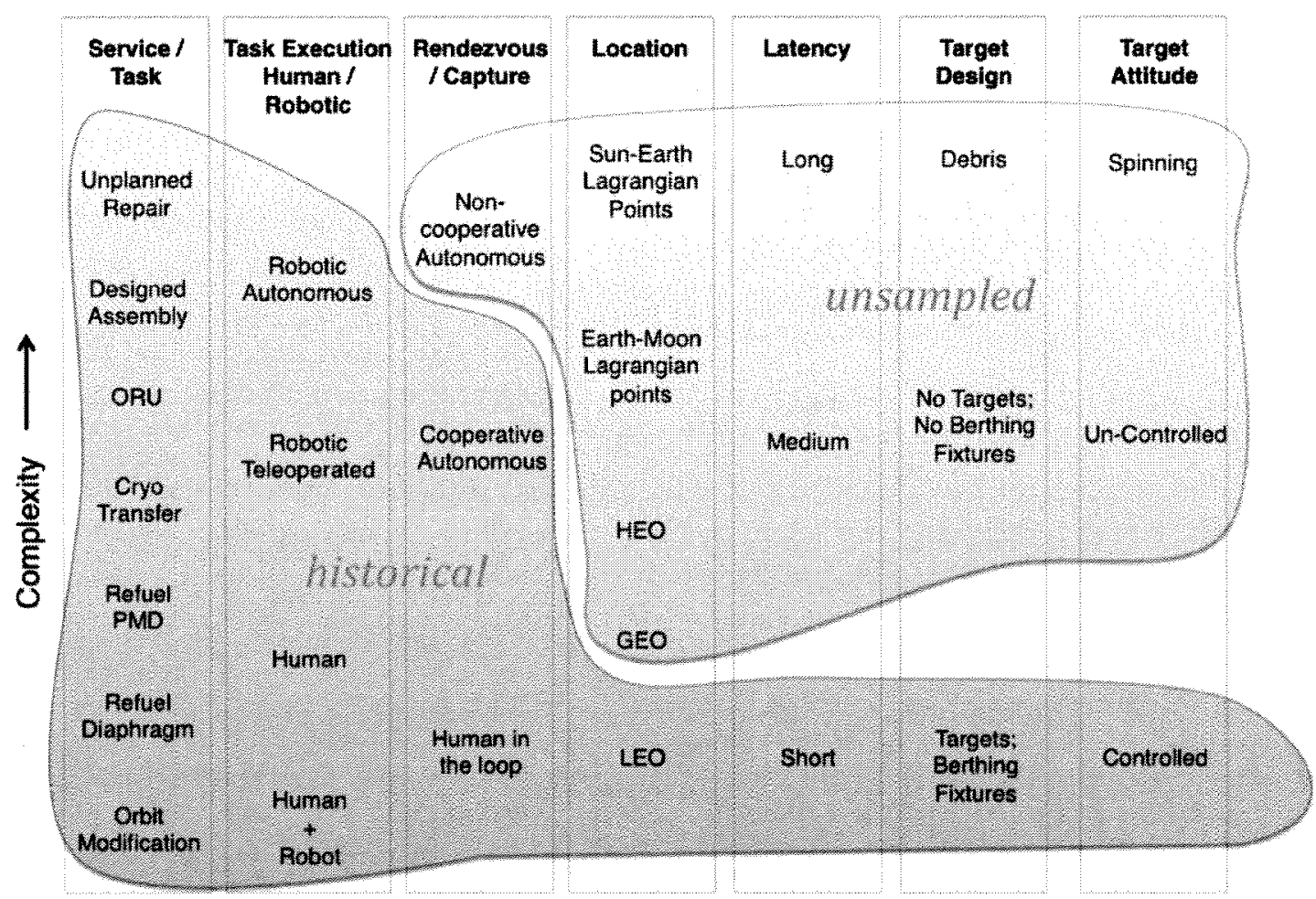

Figure 1. Servicing Study trade pace plot with historical and unsampled parameters identified.

\section{NOTIONAL MisSIONS}

The team deliberately chose not research all aspects of the satellite servicing trade space equally. To do so would have produced a study that was a 'mile wide at an inch deep,' and many studies of that type have been done. Instead we used this figure to identify where to investigate in depth; that is, studying six discrete servicing scenarios in detail in a series of design charettes. We called these servicing scenarios "notional missions" because they were deliberately designed to investigate the unsampled corners of the satellite servicing trade space in the figure. This allowed us to identify unique obstacles as well as the common elements of seemingly disparate missions.

Thus, the notional missions were chosen with the intent to explore the unique areas of the satellite servicing trade space rather than to find practical solutions for flight projects. In other words, the notional missions enabled the team to identify successful engineering solutions that could be used as data in the following analyses, rather than to produce optimized designs for a real mission.

Once the suite was identified, each of the six notional missions was developed into a proposal-level mission concept at NASA Goddard Space Flight Center's Integrated Design Center (IDC). The IDC is a collaborative, concurrent design environment. Customers come to the IDC with mission requirements and work with highly experienced 
IDC discipline engineers to create a point design mission concept to a level of detail sufficient to support a proposal. The customer determines the objectives of the assessments and shapes the 'run' to meet their needs through real-time decisions. In our case, the customers were Satellite Servicing Study team members with servicing experience from the Hubble Space Telescope Development Project.

Where possible, existing satellites and launch vehicles were employed as "customer satellites" in the design process. It is not possible to design a complete servicing mission in only one week, while simultaneously designing the customer satellite that is to be serviced. This is a salient point, as it would be easy to misinterpret our choices of customer satellites. Customer satellites were chosen solely to facilitate the design charettes and not to favor one particular customer satellite. For example, the Hubble Space Telescope was the customer satellite in the third notional mission, because it is an existing low earth-orbiting satellite and was designed to be serviced. So, its interfaces exist and are well known, which allowed the team to develop well-defined requirements for those interfaces on the notional servicer (dimensions, power, data).

The following table enumerates the high-level characteristics of each of the notional missions (NM).

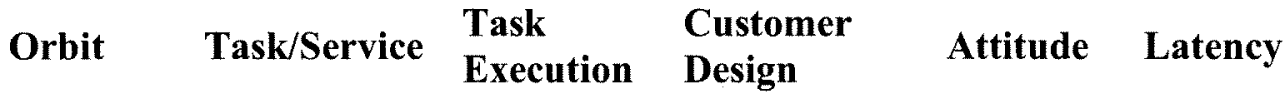

\begin{tabular}{lllllll}
\hline NM 1 & GEO & $\begin{array}{l}\text { orbit } \\
\text { modification }\end{array}$ & robots & legacy & spinning & short \\
\hline NM 2 & GEO & refueling & robots & legacy & $\begin{array}{l}\text { 3-axis } \\
\text { stabilized }\end{array}$ & short \\
\hline NM 3 & LEO & upgrade & $\begin{array}{l}\text { humans } \\
\text { (COTS) } \\
\text { robots }\end{array}$ & $\begin{array}{l}\text { designed for } \\
\text { upgrade }\end{array}$ & $\begin{array}{l}3 \text {-axis } \\
\text { stabilized }\end{array}$ & Short \\
\hline NM 4 & EML1 & assembly & robots & $\begin{array}{l}\text { designed for } \\
\text { assembly }\end{array}$ & $\begin{array}{l}\text { 3-axis } \\
\text { stabilized }\end{array}$ & medium \\
\hline NM 5 & HEO & upgrade & $\begin{array}{l}\text { humans } \\
\text { (Orion) } \\
\text { robots }\end{array}$ & $\begin{array}{l}\text { designed for } \\
\text { upgrade }\end{array}$ & $\begin{array}{l}3 \text {-axis } \\
\text { stabilized }\end{array}$ & medium \\
\hline
\end{tabular}

One major advantage of treating the notional missions as repeated design charettes with a consistent staff, assumptions, approach, and costing tools, is that the resulting engineering products are a consistent body of data from which we can make comparisons 
and draw conclusions. In addition, the six notional missions provided the Satellite Servicing team an opportunity to refine and improve the requirements at the start of the run and for the collaborating study and IDC teams to build on the insights from the previous runs. So, although the identical process was used for each notional mission, the products grew more refined with each successive notional mission run. This was both beneficial and challenging. It was challenging because in the end, the study team must understand the feasibility of servicing as well as the business case for servicing based on a set of mission studies of varying refinement. It was beneficial because the final, very complex mission studies were of such high caliber and the study team had learned so much from the IDC team that they were well equipped - in insight and in design products - to do the necessary work.

\section{COMMONALITIES}

A major objective of this study is to identify technologies and approaches that will enable satellite servicing based on a wide range of possible flight architectures. To that end, as we probed the corners of the trade space, we looked for challenges and solutions that occurred in every notional mission. Early on we identified three such areas: 1) technologies and algorithms related to rendezvous and proximity operations, 2) robotic systems, particularly those with increasing modularity and autonomy, and 3) algorithms that allow for coordinated actions of multiple spacecraft docked to one another.

By definition, servicing missions involve more than one vehicle, namely a servicer and a customer satellite in the simplest mission, with additional spacecraft in more complex missions. These spacecraft must rendezvous and mechanically join to form a 'stack'. Orbital mechanics and contact dynamics conspire to preclude the use of human teleoperated rendezvous and capture of the customer satellite. The signal latency at all orbits other than human-in-the-loop on-site (e.g., Orbiter grapple of the Hubble Space Telescope) necessitates autonomous rendezvous and capture. Therefore, successful servicing requires vehicle-relative navigation sensors, spacecraft guidance, navigation, and control algorithms, and autonomous robotic systems (remote and contact sensors, joints, local processing, algorithms). This is true no matter what architecture is used to accomplish the servicing.

Every servicing mission will include phases when multiple autonomous systems are operating simultaneously. These can include the autonomous rendezvous system, the spacecraft bus fault detection and isolation system, and the autonomous capture robotic system. During these critical phases a higher-level algorithm is required to continuously poll all stack functions for signs of trouble and manage any abort conditions. This Vehicle Autonomy Manager (VAM) algorithm will have a set of task lists at the ready so that if it receives a safing flag from any of the autonomous systems it will issue commands to the various systems updating their task lists to take the proper course of action. Consider a case where a robotic grappler had soft docked about a hardpoint on the customer satellite, but not yet hard docked and rigidized its arm joints. If, in that moment, the servicer was about to go power negative and sent a safing flag, the VAM 
would know the proper response is to command the robotic system to release the soft dock and then command the servicer G\&NC system to back away from the customer and re-orient (or slew its arrays) back to a power positive attitude.

Thus, some sort of VAM system is required for all servicing missions irrespective of the architecture that is used.

\section{CONCLUSIONS}

Our study is still underway, so conclusions at this time are interim. However, some early results include:

1. The common perception that servicing is unexplored/too difficult because of the uniqueness and complexity of servicing tasks is mistaken. The trade space diagram (Figure 1) illustrates that a very wide variety of tasks have already been conducted in-orbit. The unexplored regions in the figure include orbit location, increasing autonomy, latency, target design and attitude, but essential tasks have already been accomplished.

2. Current technologies enable multiple satellite servicing architectures at present.

3. All servicing scenarios will involve $A R \& C$ and robotics. The key variable is whether humans will be supervising and assisting via EVA, in a nearby vehicle, or from the ground.

4. If NASA's human space flight program approximates the Augustine Committee's "Flexible Path" architecture, servicing tasks that will benefit from human presence can be identified in every environment that we surveyed. Thus, a satellite servicing approach can be adapted to a variety of human flight architectures.

5. Our study did not take explicitly into account the almost-certain continued advancement of robotic and autonomous capabilities that will take place over the coming years. Servicing activities that at present appear to require astronaut intervention are likely to be taken over by robot systems in the future. 